九州大学学術情報リポジトリ

Kyushu University Institutional Repository

\title{
A Specific Difference in Growth Persistency between Oryza sativa L.. and Oryza glaberrima Steud. as Evaluated on the New Root Development Potential at Post-heading Stage.
}

Tozono, Hiromichi

Laboratory of Plant Production Physiology, Division of Soil Science and Plant Production, Department of Plant Resources, Graduate School of Bioresources and Bioenvironmental Science, Kyushu University

Hirao, Kenji

Laboratory of Plant Production Physiology, Division of Soil Science and Plant Production, Department of Plant Resources, Faculty of Agriculture, Kyushu University

Kubota, Fumitake

Laboratory of Plant Production Physiology, Division of Soil Science and Plant Production, Department of Plant Resources, Faculty of Agriculture, Kyushu University

https://doi.org/10.5109/24387

出版情報：九州大学大学院農学研究院紀要. 45 (2)，pp.387-394，2001-02-28. Kyushu University バージョン：

権利関係 : 


\title{
A Specific Difference in Growth Persistency between Oryza sativa L. and Oryza glaberrima Steud. as Evaluated on the New Root Development Potential at Post-heading Stage.
}

\author{
Hiromichi Tozono*, Kenji Hirao and Fumitake Kubota** \\ Laboratory of Plant Production Physiology, Division of Soil Science and Plant Production, \\ Department of Plant Resources, Faculty of Agriculture, Kyushu University, \\ Fukuoka 812-8581 Japan
}

(Received October 20, 2000 and accepted November 10, 2000)

\begin{abstract}
Using two strains chosen from the cultivated rice species, Oryza sativa L. and Oryza glaberrima Steud., the potential of growth persistency or growth perenniality was comparatively examined based on the photosynthate partitioning in plants grown in solution culture. The root weight of $O$. sativa strain (S-70) slightly increased for 28 days after heading; however, that of $O$. glaberrima strain (G-174) decreased. The development of new roots was restricted at the post-heading stage in both species, but the treatment of roots excision promoted new root growth, and it was more vigorous in S-70. This specific difference was more clearly shown when plants were grown at a low nitrogen concentration. Furthermore, the results of ${ }^{13} \mathrm{CO}_{2}$ feeding to plants revealed that a large amount of photosynthate was transported to stock (roots + the basal part of culm) in S-70, and the root excising treatment considerably increased the ratio of substance partitioned to stock in this species. From this evidence it may be predicted that S-70 distributes more excessive substance to roots than is required to keep their functional level during the limited maturing stage. A large accumulation of substance in stocks in S-70 may suggest that the growth perenniality has been strongly left as one of the genetic properties in $O$. sativa species.
\end{abstract}

\section{INTRODUCTION}

Oryza sativa L. and Oryza glaberrima Steud., are known as the cultivated rice species. As an ancestor species, the former has O. perennis, and the latter has $\mathrm{O}$. breviligulta (Morishima et al. 1961, 1963). Various types of growth persistency from annual to perennial are observed in $O$. perennis; however, $O$. breviligulata is regarded as a typical annual species. Both $O$. sativa and $O$. glaberrima have been cultivated as annual crop today; however, it may be predicted that a growth perenniality or trans-seasonal growth potential is considerably held in $O$. sativa, because we have frequently observed vigorous growth of new shoots or ratoons from stocks left in paddy fields after harvest. Until now some comparative studies on the growth and photosynthetic features of both species have been conducted (Sumi et al. 1991, 1994; Tagawa et al., 2000), and O. sativa was reported to remain some perenniality in growth (Kubota et al. 1992; Kawahara et al. 1997).

In general, the perennial plant is characterized by accumulating more substances in

\footnotetext{
* Laboratory of Plant Production Physiology, Division of Soil Science and Plant Production,Department of Plant Resources, Graduate School of Bioresources and Bioenvironmental Science, Kyushu University
} 
roots and culms; contrary to this, the annual plant transports the more amount of photosynthate to aboveground organs such as seeds and grains during the maturing stage. The feature of carbohydrate distribution in a plant is taken as one of the diagnotic criteria for annual and perennial growth properties. The accumulated substance is available for new shoot development in the following growth season after the end of an annual life cycle of plant. With rice plants, the partitioning of carbohydrate to roots and culms during the maturing stage is also regarded as one of the important criteria for judging the new shoot producing potential. Fushimizu et al. (1997) reported that the substance accumulation in roots and culms increased in O. sativa during the maturing stage, and the specific difference in photosynthate partitioning ratios among organs was more clearly observed at the post-heading stage (Takasaki et al. 1994).

In our study the specific difference in the potential of growth persistency between $O$. sativa and O. glaberrima was further examined and evaluated based on the change in photosynthate partition with response to root excision and fertilizing treatments conducted during the maturing stage.

\section{MATERIALS AND METHODS}

\section{Materials}

As experimental materials, Oryza sativa L. (strain No. C-70) and Oryza glaberrima Steud. (C-174) were used. Katayama (1987) collected seeds of rice species and strains from the neighboring area along the Niger River in West Africa, and the materials used in our experiment were released from his collection in Kagoshima University. In advance of the experiment, the specific characteristics in growth of both strains had been surveyed and compared for many years in our laboratory, and hence theses strains were regarded as properly selected materials for comparing the specific characteristics in growth. In this report, these two material strains were renamed S-70 for C-70 and G-174 for C-174.

\section{Cultivation conditions}

Seeds of both species were sown in nursery boxes on July 6,1999 , and young plants grown there for two weeks after germination were transplanted to two solution culture systems with different nitrogen concentrations $(1 \mathrm{~N}$ and $1 / 5 \mathrm{~N})$. These systems were placed in a vinyl house set in the Agricultural Experimental Field of Kyushu University. The standard concentration of Kimura $\mathrm{B}$ solution was used in $1 \mathrm{~N}$, and in the low nitrogen treatment $(1 / 5 \mathrm{~N})$ the nitrogen concentration was reduced to $1 / 5$ of the standard. Until these nitrogen treatments were begun, all the plants had been grown at $1 / 2 \mathrm{~N}$ solution for three weeks after transplanting. Thirty-six plants, 18 plants of $O$. sativa and $O$. glaberrima, were grown in each treatment of $1 \mathrm{~N}$ and $1 / 5 \mathrm{~N}$. Solution was renewed at 10-day intervals and the $\mathrm{pH}$ of solution was adjusted within the range of 5.0 to 5.5 every day. The solution volume in the culture system was $500 \mathrm{~L}$.

\section{Root excision treatments}

The roots of plants grown at the two nutrition levels $(1 \mathrm{~N}$ and $1 / 5 \mathrm{~N})$ were cut off at the position $2 \mathrm{~cm}$ below the culm bottom, and then the part elongated more than $2 \mathrm{~cm}$ in length in newly appeared roots was excised at two-week intervals until the 28 th day after 
heading. The heading was observed in late August, though slightly differing with species and fertilization levels. Plants were sampled at the same times with root excision treatments. The sampled plants were separated into organs such as leaf, culm, head and root, and these were dried at $80^{\circ} \mathrm{C}$ in an oven and weighed. The treatments and samplings were carried out with four replications.

\section{Measurements of leaf photosynthetic rate and dark respiratory rate of roots}

$\mathrm{CO}_{2}$ exchange rate (CER), stomatal conductance (Gs) and mesophyll conductance $(\mathrm{Gm})$ of flag leaf were measured on the 16 th day after heading, using a potable photosynthesis and transpiration measurement system (SPB-H3, ADC, UK). This measurement day fell on the second day after the second root-excision treatment. As a light source, a halogen lamp was used, and photosynthetic parameters were measured under a saturating light intensity within an air temperature range of 32 to $35^{\circ} \mathrm{C}$ in the assimilation chamber.

The entire roots of a plant were sampled, and the dark respiration rate was measured three times at two-week intervals by assimilation chamber method. The temperature during the measurement was $30 \pm 1{ }^{\circ} \mathrm{C}$, and $\mathrm{CO}_{2}$ concentration was determined with an infrared $\mathrm{CO}_{2}$ analyzer (LI-6252, Licor, USA).

\section{${ }^{13} \mathrm{CO}_{2}$ feeding and analysis of ${ }^{13} \mathrm{C}$ distribution in a plant}

Plants placed in a closed type assimilation chamber $\left(50 \times 50 \times 150 \mathrm{~cm}^{3}\right)$ covered with a permeable vinyl film were fed with ${ }^{13} \mathrm{CO}_{2}$ as substrate for photosynthesis. A dish, on which $\mathrm{Ba}^{13} \mathrm{CO}_{3}$ of $100 \mathrm{mg}$ per plant was put, was placed in the assimilation chamber and then $5 \mathrm{ml}$ of $50 \%$ phosphoric acid was added to it to produce ${ }^{13} \mathrm{CO}_{2} \cdot{ }^{13} \mathrm{CO}_{2}$ feeding was carried out for one hour under sunshine light before noon both on heading day and on the 14 th day after heading, using plants grown with and without root excision at $1 \mathrm{~N}$ and $1 / 5 \mathrm{~N}$. Three plants were measured in each treatment; 12 plants were used in total for the 4 combinations of treatments. Water mist was periodically sprayed on to the assimilation chamber surface to prevent an inadequate increase in air temperature inside.

Plant sampling was made two days after ${ }^{13} \mathrm{CO}_{2}$ feeding, and head, leaf, culm (upper and bottom parts) and root were separated and dried. The dried materials were ground, and the weight ratios of ${ }^{13} \mathrm{C}$ to ${ }^{12} \mathrm{C}$ in pulverized matters were measured with a ${ }^{13} \mathrm{C}$ analyzer (EX-130, Nihonbunko, Japan). Based on the measurements obtained, ratios of photosynthate partitioning to different organs and plant parts were calculated.

\section{RESULTS AND DISCUSSION}

Fig. 1 shows the time courses of shoot and root weights in both species to compare the effects of root excision and fertilization treatments on them. Shoots of plants without root excision showed a vigorous growth in G-174, particularly when it was grown at $1 \mathrm{~N}$. The unexcised plants of $\mathrm{G}-174$ had a larger weight of roots as a whole, but the root weight decreased linearly with maturity whereas it showed an increasing tendency in S-70. The substance accumulated in the annual plant is known to quickly travel into the reproductive organ during the maturing stage; such a phenomenon was more conspicuously found in G-174. A higher sensitivity in dry matter production to 

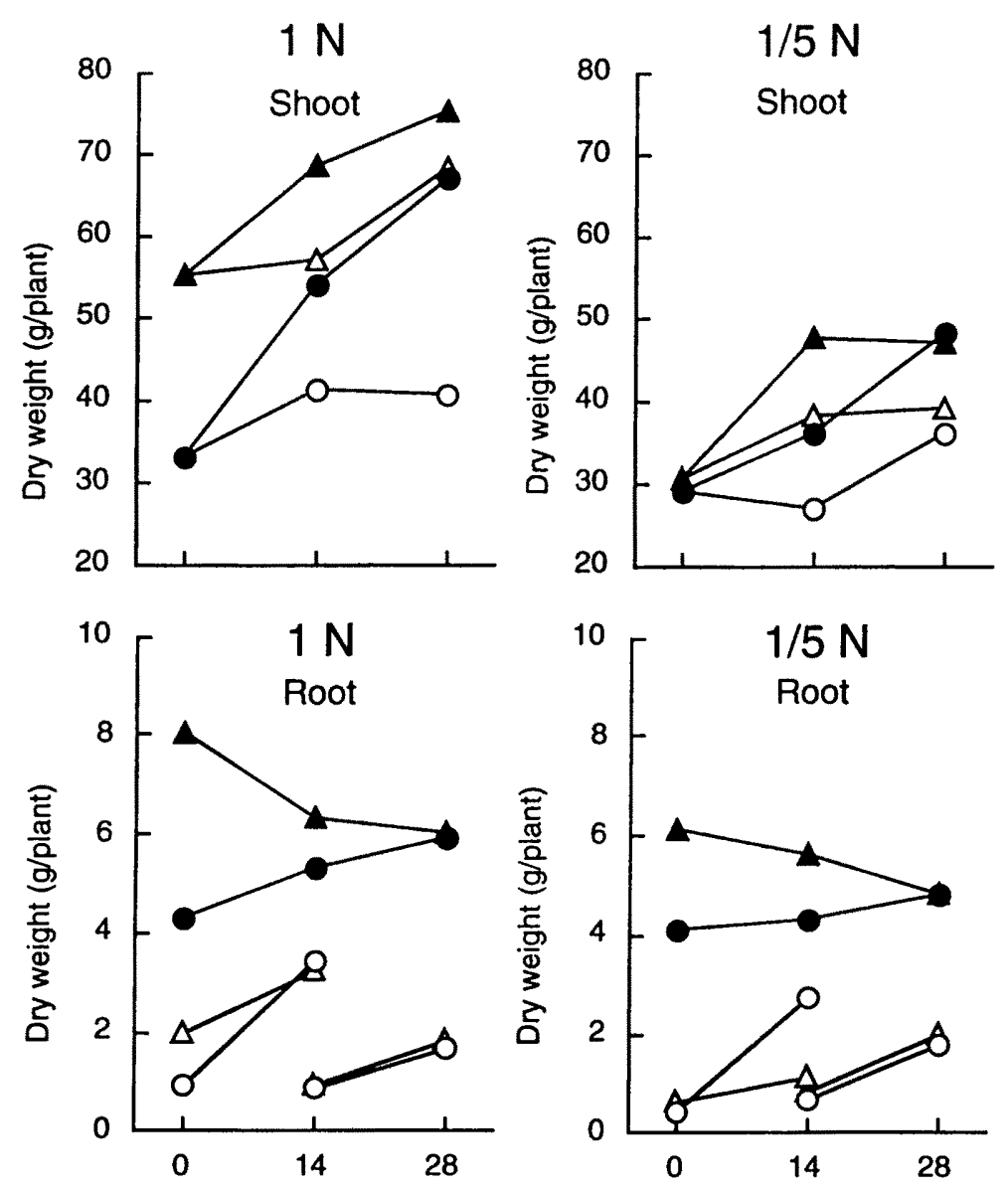

Days after heading

Days after heading

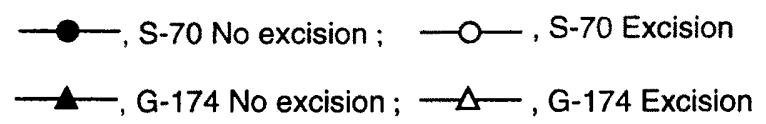

Fig. 1. Effects of root excision and fertilization on dry matter weight of shoots and roots in S-70 and G-174.

fertilization was observed in O. glaberrima (Sumi et al., 1991; Kubota et al., 1992), and such a specific feature is recognized here as well.

The roots of $\mathrm{G}-174$ plant grown without root excision decreased in weight after heading as mentioned above, but after roots were excised at heading and 14 days after heading the root weight increased at both $1 \mathrm{~N}$ and $1 / 5 \mathrm{~N}$ (Fig. 1). Like this, the root excision treatment is able to promote new root development and growth in both species, 
and root weight increase by the excision treatment was more clearly shown in S-70, particularly in the plant grown in $1 / 5 \mathrm{~N}$.

Table 1 shows the combined effects of root excision and fertilization on CER and related parameters in G-174 and S-70 measured directly before the second root excision, which corresponded to the 14 th day after heading. As compared between both strains

Table 1. Effects of root excision and fertilization treatments on leaf photosythetic parameters.

\begin{tabular}{ccccccc}
\hline & & \multicolumn{2}{c}{$1 \mathrm{~N}$} & & \multicolumn{2}{c}{$1 / 5 \mathrm{~N}$} \\
\cline { 3 - 4 } \cline { 6 - 7 } & & Not excised & Excised & & No excised & Excised \\
\hline CER & $\mathrm{S}-70$ & $22.05^{\mathrm{a}}$ & $19.50^{\mathrm{b}}$ & & $11.92^{\mathrm{a}}$ & $11.88^{\mathrm{a}}$ \\
$\left(\mu \mathrm{molm}^{-2} \mathrm{~s}^{-1}\right)$ & $\mathrm{G}-174$ & $16.98^{\mathrm{c}}$ & $12.40^{\mathrm{d}}$ & & $13.10^{\mathrm{a}}$ & $11.27^{\mathrm{a}}$ \\
\hline $\mathrm{Gs}$ & $\mathrm{S}-70$ & $0.38^{\mathrm{a}}$ & $0.34^{\mathrm{ab}}$ & & $0.22^{\mathrm{a}}$ & $0.21^{\mathrm{a}}$ \\
$\left(\mathrm{molm}^{-2} \mathrm{~s}^{-1}\right)$ & $\mathrm{G}-174$ & $0.41^{\mathrm{a}}$ & $0.29^{\mathrm{b}}$ & & $0.41^{\mathrm{b}}$ & $0.35^{\mathrm{b}}$ \\
\hline $\mathrm{Gm}$ & $\mathrm{S}-70$ & $0.097^{\mathrm{a}}$ & $0.084^{\mathrm{ab}}$ & & $0.051^{\mathrm{a}}$ & $0.054^{\mathrm{a}}$ \\
$\left(\mathrm{molm}^{-2} \mathrm{~s}^{-1}\right)$ & $\mathrm{G}-174$ & $0.065^{\mathrm{c}}$ & $0.048^{\mathrm{d}}$ & & 0.050 & $0.044^{\mathrm{a}}$ \\
\hline
\end{tabular}

$1 \mathrm{~N}$, standard concentration of Kimura B solution;

$1 / 5 \mathrm{~N}$, nitrogen concentration was reduced to $1 / 5$ of the standard Kimura B solution.

Values followed by the same letter are not significantly different at $5 \%$ level.
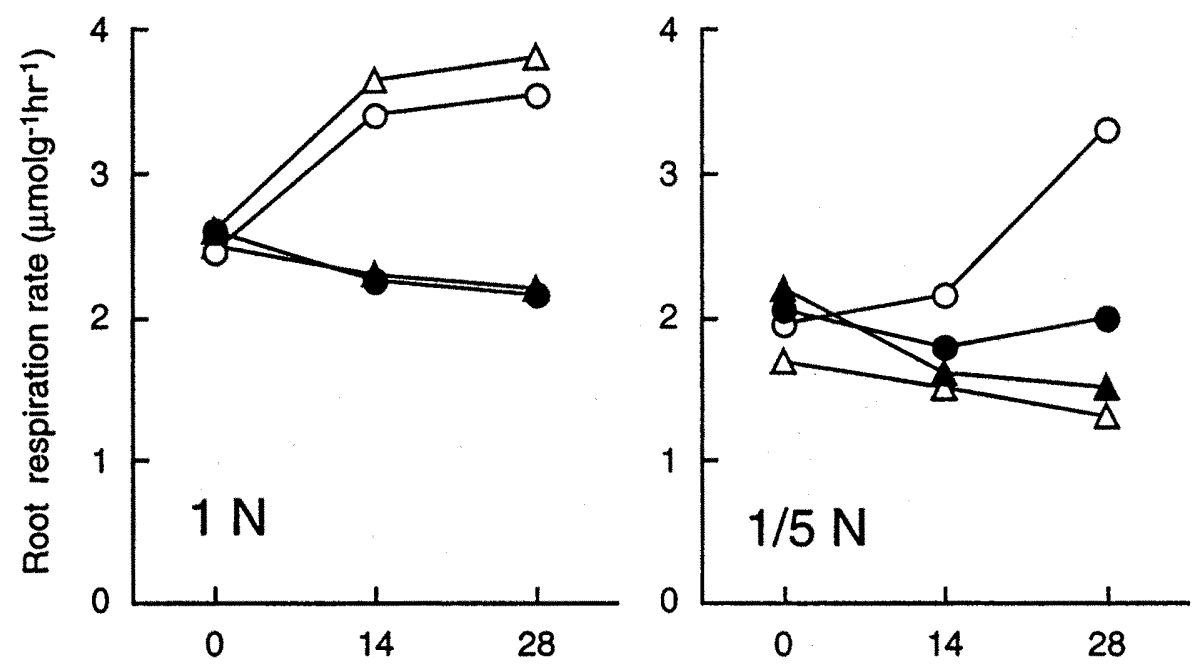

Days after heading

Fig. 2. Effects of root excision and fertilization on the root respiration rate in S-70 and G-174. See Fig. 1 for the simbols. 
grown without root excision, CER measured at $1 \mathrm{~N}$ was significantly higher in S-70 than in G-174. However, there was not a significant difference between CERs in both strains grown at $1 / 5 \mathrm{~N}$. CER of plants subjected to root excisions was also significantly higher in S-70 compared to that of G-174. Contrary to this, Gs showed high values in G-174 as a whole, and it was not decreased by root excision and also by reduced nitrogen treatment. Like this, there is a specific feature in stomatal and photosynthetic responses to

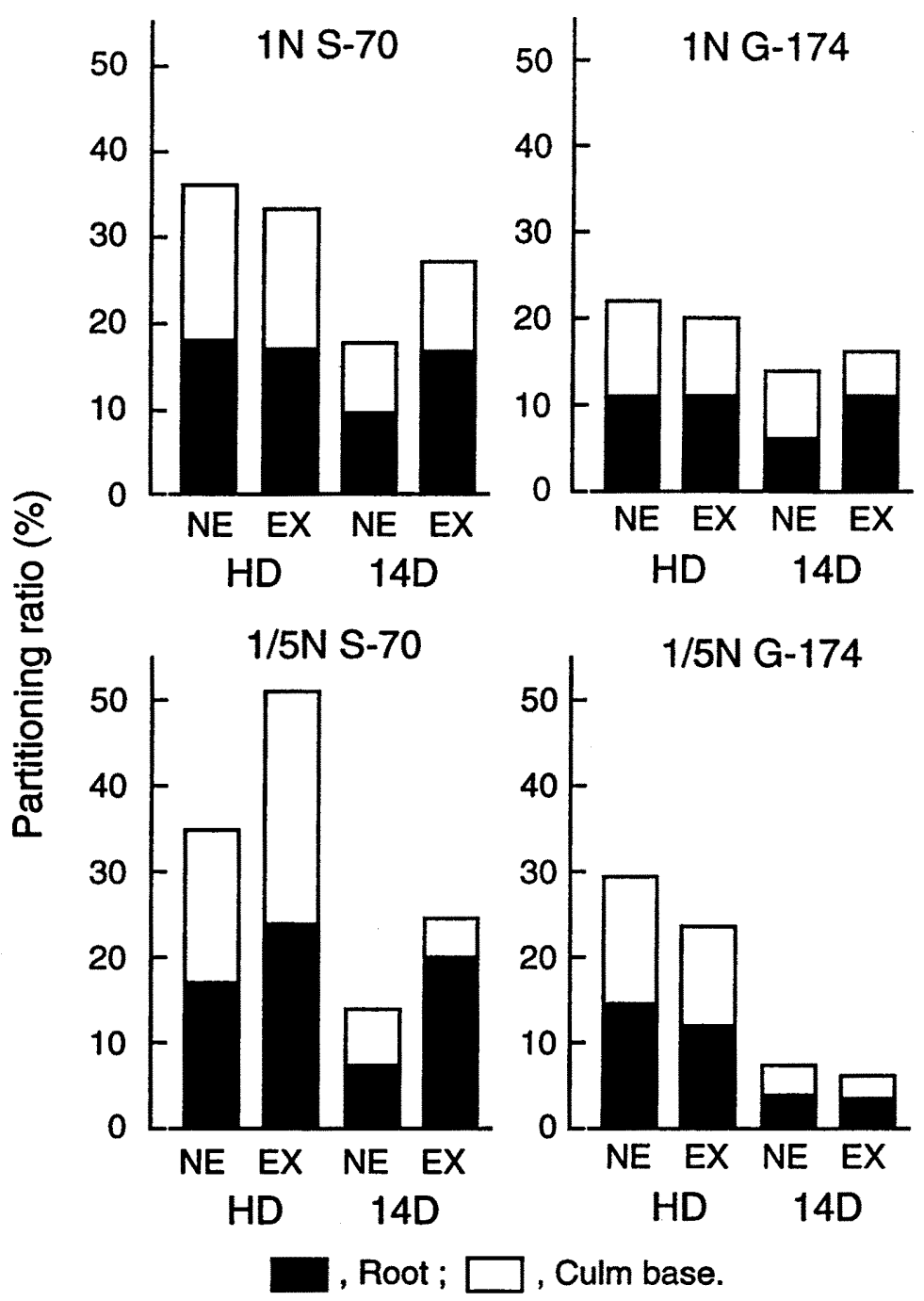

Fig. 3. The partitioning ratio of ${ }^{13} \mathrm{C}$ to stock (root + culm base) at heading day (HD) and the 14 th day after heading (14D) in S-70 and G-174. The percentage shows the ratio to the total ${ }^{13} \mathrm{C}$ weight containing in a whole plant. NE, no root excision; $\mathrm{EX}$, root excision. 
treatments.

CER and Gm were reduced by root excisions in both species, and these reductions were more conspicuous in G-174. A large depression of these parameters in G-174 is likely due to the insufficient recovery of root functions after root excision. As shown in Fig. 1, G-174 grown without root excision featured a more vigorous growth in shoots and roots, but the result of root excision treatment may suggest that the activity of transporting substance to roots in G-174 was inferior to that of S-70. On the other hand, the root excision did not offer so strong effects on these two parameters in plants grown at $1 / 5 \mathrm{~N}$. As a reason for this, it may be considered that plants grown at mal-nutrient conditions are not vigorous in growth and this results in reducing the responsiveness to root excision.

Fig. 2 shows the effect of root excision on dark respiration rate of roots in G-174 and $\mathrm{S}-70$. In plants grown without root excision at $1 \mathrm{~N}$, the root respiration rate decreased with time after heading; contrary to this, the rate in plants subjected to root excision increased. This fact is likely caused by the development of new active roots after root cutting. There was not significant difference in respiration rate between both strains grown at $1 \mathrm{~N}$, whereas an inter-specific difference was found in plants grown at $1 / 5 \mathrm{~N}$. The root respiration rate of S-70 was increased by root excision, but not in G-174. S-70 is considered to be capable of keeping a higher potential of photosynthate partitioning to roots even under mal-nutrient growth condition. For the plants grown without root excision at $1 / 5 \mathrm{~N}$, a higher dark respiration was also observed in $\mathrm{S}-70$, but it was not significantly different from that of G-174.

Based on ${ }^{13} \mathrm{C}$ accumulation weight in each organ, the ratio of photosynthate partitioning to stock (roots + culm base) was calculated. The effect of root excision and fertilization on the partitioning ratio is revealed in Fig. 3. The culm base is defined here as a bottom $5 \mathrm{~cm}$ part in culm. The partitioning ratio of stocks was considerably high in S-70, particularly in the case that ${ }^{13} \mathrm{CO}_{2}$ was fed at heading day. An interesting point is that the partitioning ratio was higher in $\mathrm{S}-70$ grown with root excision at $1 / 5 \mathrm{~N}$. Like this, S-70 is characterized by maintaining the accumulation of substance in stocks during maturing stage even under such a growth condition as limited nitrogen application. This growth feature is beneficial in enhancing and maintaining new shoot producing potential toward the following growth season. It may be concluded through the combined treatments of root excision and fertilization that the annual and perennial growth features were more clearly recognized in both strains of $O$. glaberrima and $O$. sativa, respectively.

\section{LITERATURES}

Fushimizu K., K. Hirao, K. Saitou, F. Kubota and W. Agata 1997 Comparison for the growth perenniality between Africa-grown rice cultivars, Oryza sativa L. and Oryza glaberrima Steud. Sci. Bull. Agr. Kyushu Univ., 51: 125-130

Katayama T. C. 1987 General remarks on cultivated rice in Africa concerned. Kagoshima Univ. Res. Center S. Pac. Occasional Papers, 10: 91-102

Kawahara T., F. Kubota, K. Hirao, K. Saitou and W. Agata 1998 Interspecific difference in growth perenniality in rice cultivars, Oryza sativa L. and O. glaberrima Steud. J. Fac. Agr. Kyushu Univ., 42: $315-323$

Kubota F., S. Okano, W. Agata and T. C. Katayama 1992 Responses of dry matter production and 
photosynthesis in Oryza glaberrima Steud. and Oryza sativa L. introduced from West Africa to the concentrations of culture solution. Jpn. J. Crop Sci., 61: 207-212

Morishima H., H. I. Oka and W. T. Chang 1961 Directions of differentiation in populations of wild rice, Oryza perennis and $O$. sativa f. spontanea. Evolution 15: 326-339

Morishima H., K. Hinata and H. I. Oka 1963 Comparison of modes of evolution of cultivated forms from two wild rice species, Oryza breviligulata and O. perennis. Evolution 17: 170-181

Sumi A. and T. C. Katayama 1991 Dry matter increase and water consumption of rice plants collected in Senegal; comparison of Oryza sativa L. and Oryza glaberrima Steud. Rep. Kyushu Br.Crop Sci. Soc. Jpr. 58: 73-76

Sumi A., T. C. Katayama and W. Agata 1994 Studies on agronomic traits African rice (Oryza glaberrima Steud.) II. Dry matter increase and water use efficiency. Jpn. J. Crop Sci, 63: 105-110

Tagawa T., K. Hirao and F. Kubota 2000 A specific feature of nitrogen utilization efficiency in leaf photosynthesis in Oriza glaberrima Steud. Jpn. J. Crop Sci. 69: 74-79

Yoon Y. H., A. Isoda, H. Nojima and Y. Takasaki 1998 Differences in growth and translocation after heading between two strains of Oryza glaberrima Steud. and two cultivars of Oryza sativa L. Jpn. J. Crop Sci., 67: 379-383 